\title{
Cancer risk in patients aged 30 years and above with type 2 diabetes receiving antidiabetic monotherapy: a cohort study using metformin
} as the comparator

\author{
This article was published in the following Dove Press journal: \\ Therapeutics and Clinical Risk Management \\ 28 August 2015 \\ Number of times this article has been viewed
}

\author{
Yu-Ching Chen' \\ Victor C Kok ${ }^{1,2}$ \\ Ching-Hsuan Chien' \\ Jorng-Tzong Horngl,3 \\ Jeffrey J P Tsai' \\ 'Department of Biomedical \\ Informatics, Asia University, Taichung, \\ ${ }^{2}$ Department of Internal Medicine, \\ Kuang Tien General Hospital, Taichung, \\ ${ }^{3}$ Department of Computer Science \\ and Information Engineering, National \\ Central University, Jhongli, Taiwan
}

Correspondence: Victor C Kok Department of Internal Medicine, Kuang Tien General Hospital, I 17 Shatien Rd, Shalu, Taichung 43303, Taiwan Email victorkok@asia.edu.tw

\section{Yu-Ching Chen}

Department of Biomedical Informatics, Asia University Taiwan, 500 Lioufeng Rd, Wufeng, Taichung 4I354, Taiwan Email yuching@asia.edu.tw
Introduction: Accumulating evidence suggests that metformin reduces incident cancer development. Few cohort studies have evaluated the risk of subsequent cancer development in diabetic cohorts receiving antidiabetic monotherapy. We conducted a population-based study in patients with new-onset type 2 diabetes treated with antidiabetic monotherapy.

Methods: We identified a cohort of patients with type 2 diabetics aged $\geq 30$ years receiving hypoglycemic monotherapy $(n=7,325)$ from the 1998-2007 Longitudinal Health Insurance Dataset. Patients were grouped according to the antidiabetic therapy they received into metformin $(n=2,223)$, sulfonylurea $(n=3,965)$, glitazone $(n=53)$, meglitinide $(n=128)$, acarbose $(n=150)$, and insulin $(n=806)$ groups. Patients with preexisting cancer were excluded. All patients were followed up until cancer development, dropout, death, or until December 31, 2008. Cox's model was used to estimate multivariable hazard ratios (HRs) adjusted for age, sex, Charlson comorbidity index, smoking-related comorbidities, alcohol use disorders, morbid obesity, pancreatitis, hypertension, monthly income, and urbanization level. The log-rank test was used to compare cumulative cancer incidence. Two-sided $P$-values $<0.05$ were required to reject the null hypothesis.

Results: The overall median follow-up duration was 2.5 years (interquartile range, 3.6 years). Totally, 367 and 124 cancers developed in the sulfonylurea and metformin groups, respectively, representing an adjusted HR of 1.36 (95\% confidence interval $[\mathrm{CI}], 1.11-1.67 ; P<0.005)$. No significant differences were observed between other groups. Increased adjusted HRs were observed for colorectal cancer (adjusted HR, 1.94; 95\% CI, 1.15-3.27; $P<0.05$ ) and lung cancer (adjusted HR, 1.76; 95\% CI, 1.00-3.07; $P<0.05$ ).

Conclusion: Metformin monotherapy may be associated with a reduction in the risk for cancer development compared with sulfonylurea monotherapy. Moreover, the use of an average defined daily dose of $>0.25$ of metformin when compared to lower dose will contribute to a reduction of $80 \%$ risk.

Keywords: type 2 diabetes, antidiabetic drug, monotherapy, metformin, sulfonylureas, cancer risk, NHIRD

\section{Introduction}

The number of adults aged $\geq 25$ years afflicted with diabetes mellitus (DM) worldwide was estimated to be over 347 million in 2008. ${ }^{1}$ Type 2 diabetes per se portends an increased risk of future cancer development, particularly colorectal, breast, liver, pancreas, urinary tract, and female reproductive organ cancers. ${ }^{2}$ The initial management of new-onset diabetes includes providing education on lifestyle interventions 
and behavioral modifications. Single hypoglycemic agents are used in cases where glycemic control remains suboptimal. A widely accepted consensus algorithm for the initiation of therapy in patients with newly diagnosed type 2 diabetes was copublished by the American Diabetes Association and the European Association for the Study of Diabetes. ${ }^{3}$

Accumulating evidence from epidemiologic studies suggests that the use of metformin (dimethylbiguanide) as an antidiabetic agent is associated with a reduced risk of incident cancer development in the future. ${ }^{4-8}$ Metformin requires the Peutz-Jeghers protein, LKB1, in the liver to reduce gluconeogenesis. LKB1 is a tumor suppressor that phosphorylates and activates adenosine monophosphate-activated protein kinase (AMPK) to inhibit cell growth. ${ }^{9}$ Nevertheless, perhaps an even greater number of studies have suggested a neutral effect of metformin on the risk of future cancer development. ${ }^{10-17}$ Given the current evidence, there remains a lack of consensus regarding the beneficial effect of metformin on cancer risk. In the last year, a large retrospective cohort study in the United Kingdom Clinical Practice Research Datalink analyzed in the manner of an intention-to-treat trial concluded that individuals with diabetes using metformin $(n=51,484 ; 54 \%$ of total participants) had a similar risk of developing cancer compared with those using sulfonylureas $(n=18,264 ; 19 \%$ of total participants). ${ }^{18}$ This issue is further complicated by the frequent use of combination therapies (at least more than one hypoglycemic agent) or the subsequent requirement of crossover treatments for glycemic control.

There have been few cohort studies examining the risk of subsequent cancer development in diabetic patients receiving prolonged hypoglycemic monotherapy. Therefore, we conducted a population-based retrospective cohort study in patients with newly diagnosed type 2 diabetes treated with antidiabetic pharmacological monotherapy.

\section{Methods}

\section{Study design and data source}

We designed a population-based retrospective cohort study in a diabetic cohort of 76,082 individuals using the Longitudinal Health Insurance Dataset (LHID), a database constructed for research purposes from the original registration files and original claim data in the Taiwan National Health Insurance Research Database (NHIRD).

The National Health Insurance (NHI) program in Taiwan was launched in 1995 . In 2005, the program covered $98 \%$ of all residents in Taiwan. The NHIRD is a comprehensive database containing four major database files: ambulatory care expenditures by visits, inpatient expenditures by admissions, details of ambulatory care orders, and details of inpatient order files. The LHID comprises data of 1 million participants randomly sampled from the NHIRD. No statistically significant difference in sex distribution was observed between patients in the LHID and NHIRD $\left(\chi^{2}=0.067\right.$, degree of freedom $[d f]=1, P=0.796)$. The authors have published over 20 population-based cohort studies using either the LHID or NHIRD. ${ }^{19-22}$

\section{Ethics statement}

The NHIRD data files were anonymized by scrambling the identification codes of both individuals and medical institutions. This study met the exemption criteria for review by the Institutional Review Board (IRB) and was approved by the Kuang Tien General Hospital accredited in-house IRB, with an approval number of KTGH-10429. This study strictly adhered to confidentiality guidelines in accordance with the regulations set forth by the Personal Information Protection Act of Taiwan and was conducted in accordance with the Declaration of Helsinki.

\section{Selection of patients and data extraction}

The target population for this study was patients with newonset type 2 diabetes aged $\geq 30$ years receiving a single hypoglycemic drug (monotherapy) for glycemic control without preexisting cancer at the index date. Patients who subsequently crossed over to a different hypoglycemic drug or initiated the use of combination therapy were excluded.

For case and disease definitions, at least three outpatient claims or one inpatient claim using the International Classification of Disease, Ninth Revision, Clinical Modification (ICD-9-CM) code were required. The diagnostic criterion for type 2 diabetes in this cohort was fasting plasma glucose level $\geq 126 \mathrm{mg} / \mathrm{dL}$ ( $7 \mathrm{mmol} / \mathrm{L}$ ). Patients with any previous claim for a diagnosis of cancer or corresponding treatment, in either inpatient or outpatient settings, were excluded because of preexisting cancer.

Data for each eligible participant, including age, sex, smoking-related diagnoses (ICD-9-CM code 305.1, 491.2, 492.8, 496, 523.6, 959.84, 649.0, and V15.82), alcohol use disorders $(265.2,291,303,305.0,357.5,425.5,626.3,571.0$, $571.1,571.2,571.3,980.0$, and V11.3), morbid obesity (278, 646.1, 649.1, 649.2, V45.86, V65.3, and V77.8), pancreatitis (577.0 and 577.1), hypertension (410-414), hyperlipidemia (272), monthly household income as a proxy of socioeconomic status (four levels according to monthly insurance premiums: less than NTD \$19,999, \$20,000-\$39,999, $\geq \$ 40,000$, and missing data), and urbanization level (five strata from the 
least to the most) were extracted for analysis. The Charlson comorbidity index (CCI) was used to reveal the medical comorbidities of each participant.

\section{Identification of study cohorts}

Figure 1 shows the method used to identify study cohorts. In the 1998-2007 LHID ( $n=959,982)$, a cohort of enrollees with DM was identified $(n=76,082)$. From this cohort of patients with diabetes, 7,399 were excluded because of preexisting DM (ICD-9-CM code 250.xx), 4,298 were excluded because of type 1 diabetes (ICD-9-CM code 250.x1 or 250.x3), 1,390 were excluded because of being younger than 30 years of age, 12,307 were excluded because of preexisting cancer (ICD-9-CM codes 140-209 [frank malignancy]; 230-234 [in situ cancer]), and 24 were excluded because of unknown sex. As a result, the data of 50,664 patients with new-onset type 2 diabetes were extracted. Patients were screened for the use of antidiabetic monotherapy throughout the study period, resulting in the exclusion of 43,339 patients who failed to meet the criteria. In other words, real-world data demonstrate that the proportion of patients aged $\geq 30$ years with type 2 diabetes and receiving prolonged monotherapy in Taiwan is $14.46 \%(7,325 / 50,664 \times 100 \%)$.

During the study period, six hypoglycemic agents were reimbursed by the NHI, namely, biguanides (only metformin available); glitazones (also known as thiazolidinediones), including rosiglitazone and pioglitazone; sulfonylureas, including acetohexamide, chlorpropamide, tolbutamide, tolazamide, glipizide, gliclazide, glyburide (also known as glibenclamide), glibornuride, gliquidone, and glimepiride; meglitinides, including repaglinide and nateglinide; alpha-glucosidase inhibitors (only acarbose available); and insulin. The entire target population was then grouped according to the type of hypoglycemic drug used into sulfonylurea $(n=3,965 ; 54.1 \%)$, metformin $(n=2,223 ; 30.3 \%)$, insulin $(n=806 ; 11 \%)$, acarbose $(n=150 ; 2 \%)$, meglitinide $(n=128 ; 1.8 \%)$, and glitazone $(n=53 ; 0.7 \%)$ groups. Table 1 lists the demographic data at baseline grouped according to the six hypoglycemic monotherapy groups.

The medication indications for selecting an antidiabetic monotherapy in the studied period of 1998-2007 in this region are as follows: sulfonylurea used to be the first-line treatment



Figure I Consort diagram demonstrating the patient selection process.

Abbreviations: LHID, Longitudinal Health Insurance Dataset; DM, diabetes mellitus. 
Table I Demographic data for newly diagnosed diabetic patients according to antidiabetic monotherapy

\begin{tabular}{|c|c|c|c|c|c|c|c|}
\hline Hypoglycemic & Metformin & Glitazone & Sulfonylurea & Meglitinide & Acarbose & Insulin & Total \\
\hline $\mathrm{N}(\%)$ & 2,223 & 53 & 3,965 & 128 & 150 & 806 & 7,325 \\
\hline \multicolumn{8}{|l|}{ Age (year) } \\
\hline Median (IQR)** & $60.6(19.5)$ & $61.0(21.2)$ & $62.4(19.9)$ & $67.2(23.4)$ & $63.5(22.6)$ & $70.6(22.5)$ & $62.6(20.4)$ \\
\hline $30-39$ & $128(5.8)$ & $3(5.7)$ & $177(4.5)$ & $5(3.9)$ & $12(8.0)$ & $57(7.1)$ & $382(5.2)$ \\
\hline $40-49$ & $359(16.1)$ & $7(13.2)$ & $667(16.8)$ & $12(9.4)$ & $17(11.3)$ & $75(9.3)$ & $\mathrm{I}, \mathrm{I} 37$ (I5.5) \\
\hline $50-59$ & $598(26.9)$ & $15(28.3)$ & 931 (23.5) & $34(26.6)$ & $36(24.0)$ & III (I3.8) & I,725 (23.5) \\
\hline $60-69$ & $54 \mid(24.3)$ & $10(18.9)$ & $1,018(25.7)$ & $21(16.4)$ & $37(24.7)$ & $143(17.7)$ & I,770 (24.2) \\
\hline $70-79$ & $469(21.1)$ & $16(30.2)$ & $864(21.8)$ & $31(24.2)$ & $37(24.7)$ & $234(29.0)$ & $\mathrm{I}, 65 \mathrm{I}(22.5)$ \\
\hline$\geq 80$ & $128(5.8)$ & $2(3.8)$ & $308(7.8)$ & $25(19.5)$ & II (7.3) & $186(23.1)$ & $660(9.0)$ \\
\hline \multicolumn{8}{|l|}{ Sex* } \\
\hline Woman & $\mathrm{I}, 028(46.2)$ & $21(39.6)$ & $1,730(43.6)$ & $53(41.4)$ & $82(54.7)$ & $349(43.3)$ & $3,263(44.5)$ \\
\hline Man & $\mathrm{I}, 195(53.8)$ & $32(60.4)$ & $2,235(56.4)$ & $75(58.6)$ & $68(45.3)$ & 457 (56.7) & $4,062(55.5)$ \\
\hline \multicolumn{8}{|l|}{$\mathrm{CCl}$} \\
\hline Median (IQR)*** & $1.0(2.0)$ & $1.0(2.0)$ & $1.0(2.0)$ & $\mathrm{I} .0(2.0)$ & $\mathrm{I} .0(2.0)$ & $2.0(3.0)$ & $1.0(2.0)$ \\
\hline 0 & $\mathrm{I}, 038(46.7)$ & $24(45.3)$ & $1,600(40.4)$ & $57(44.5)$ & 61 (40.7) & $123(15.3)$ & $2,903(39.6)$ \\
\hline 1 & $609(27.4)$ & II (20.8) & $\mathrm{I}, 002(25.3)$ & $27(21.1)$ & $4 I(27.3)$ & $161(20.0)$ & $\mathrm{I}, 85 \mathrm{I}(25.3)$ \\
\hline 2 & 297 (I 3.4$)$ & $10(18.9)$ & $578(14.6)$ & $16(12.5)$ & $22(14.7)$ & $138(17.1)$ & $\mathrm{I}, 06 \mathrm{I}(\mathrm{I} .5)$ \\
\hline 3 & $134(6.0)$ & $3(5.7)$ & $336(8.5)$ & $7(5.5)$ & $14(9.3)$ & $131(16.3)$ & $625(8.5)$ \\
\hline 4 & $70(3.1)$ & $\mathrm{I}(1.9)$ & $219(5.5)$ & II (8.6) & $5(3.3)$ & $89(11.0)$ & $395(5.4)$ \\
\hline 5 & $4 \mid(1.8)$ & $3(5.7)$ & $105(2.6)$ & $3(2.3)$ & $5(3.3)$ & $68(8.4)$ & $225(3.1)$ \\
\hline 6 & $20(0.9)$ & I (I.9) & $69(1.7)$ & $5(3.9)$ & I (0.7) & $38(4.7)$ & $131(1.8)$ \\
\hline$\geq 7$ & $14(0.6)$ & $0(0.0)$ & $56(1.4)$ & $2(1.6)$ & $\mathrm{I}(0.7)$ & $58(7.2)$ & $134(1.8)$ \\
\hline Smoking-related diagnoses** & $295(13.3)$ & $8(15.1)$ & $656(16.5)$ & $18(14.1)$ & $15(10.0)$ & $213(26.4)$ & $\mathrm{I}, 205(16.5)$ \\
\hline Alcohol use disorder** & $48(2.2)$ & I (I.9) & $120(3.0)$ & $5(3.9)$ & $4(2.7)$ & $54(6.7)$ & $232(3.2)$ \\
\hline Morbid obesity** & $70(3.1)$ & $2(3.8)$ & $33(0.8)$ & $\mathrm{I}(0.8)$ & $6(4.0)$ & $3(0.4)$ & $115(1.6)$ \\
\hline Pancreatitis ${ }^{* *}$ & II (0.5) & $0(0.0)$ & $41(1.0)$ & $3(2.3)$ & $3(2.0)$ & $44(5.5)$ & $102(1.4)$ \\
\hline Hypertension & $\mathrm{I}, 447(65 . \mathrm{I})$ & $39(73.6)$ & $2,544(64.2)$ & $83(64.8)$ & $93(62.0)$ & $480(59.6)$ & $4,686(64.0)$ \\
\hline Hyperlipidemia ${ }^{* *}$ & $\mathrm{I}, 133(5 \mathrm{I} .0)$ & $27(50.9)$ & $\mathrm{I}, 756(44.3)$ & $55(43.0)$ & $67(44.7)$ & $161(20.0)$ & $3,199(43.7)$ \\
\hline \multicolumn{8}{|l|}{ Monthly income in NTD** } \\
\hline $1-19,999$ & $\mathrm{I}, 450(65.2)$ & $29(54.7)$ & $2,853(72.0)$ & $99(77.3)$ & $103(68.7)$ & $606(75.2)$ & $5,140(70.2)$ \\
\hline $20,000-39,999$ & $349(15.7)$ & II (20.8) & $459(11.6)$ & $8(6.2)$ & $19(12.7)$ & $48(6.0)$ & $894(12.2)$ \\
\hline$\geq 40,000$ & $205(9.2)$ & $8(15.1)$ & $239(6.0)$ & $5(3.9)$ & $8(5.3)$ & $29(3.6)$ & $494(6.7)$ \\
\hline Missing data & $219(9.9)$ & $5(9.4)$ & $414(10.4)$ & $16(12.5)$ & $20(13.3)$ & $123(15.3)$ & $797(10.9)$ \\
\hline \multicolumn{8}{|l|}{ Urbanization level** } \\
\hline Most urbanized & $664(29.9)$ & $17(32.1)$ & $996(25.1)$ & $32(25.0)$ & $30(20.0)$ & $192(23.8)$ & I,93I (26.4) \\
\hline More & $652(29.3)$ & $16(30.2)$ & $\mathrm{I}, 084(27.3)$ & $29(22.7)$ & $45(30.0)$ & $200(24.8)$ & $2,026(27.7)$ \\
\hline Moderate & $368(16.6)$ & $9(17.0)$ & $631(15.9)$ & $20(15.6)$ & $22(14.7)$ & $148(\mid 8.4)$ & $\mathrm{I}, 198(16.4)$ \\
\hline Less & $289(13.0)$ & $6(11.3)$ & $685(17.3)$ & $20(15.6)$ & $30(20.0)$ & $133(16.5)$ & I,I 63 (I5.9) \\
\hline Least & $250(11.2)$ & $5(9.4)$ & $569(14.4)$ & $27(21.1)$ & $23(15.3)$ & $133(16.5)$ & $\mathrm{I}, 007(\mathrm{I} 3.7)$ \\
\hline \multicolumn{8}{|l|}{ Duration of follow-up (years) } \\
\hline Median (IQR)** & $2.4(2.9)$ & $2.8(2.8)$ & $2.8(3.9)$ & $2.8(3.5)$ & $2.1(2.7)$ & $1.6(3.7)$ & $2.5(3.6)$ \\
\hline$\leq 3$ & $\mathrm{I}, 345(60.5)$ & $28(52.8)$ & $2,104(53.1)$ & $92(71.9)$ & $92(61.3)$ & $535(66.4)$ & $4,196(57.3)$ \\
\hline$>3$ to $\leq 5$ & $473(21.3)$ & $19(35.8)$ & $829(20.9)$ & $23(18.0)$ & $40(26.7)$ & $112(13.9)$ & $\mathrm{I}, 496(20.4)$ \\
\hline$>5$ to $\leq 7$ & $198(8.9)$ & $6(11.3)$ & $482(12.2)$ & II (8.6) & $18(12.0)$ & $60(7.4)$ & $775(10.6)$ \\
\hline$>7$ to $\leq 9$ & $154(6.9)$ & $0(0.0)$ & $373(9.4)$ & $2(1.6)$ & $0(0.0)$ & $53(6.6)$ & $582(7.9)$ \\
\hline$>9$ & $53(2.4)$ & $0(0.0)$ & $177(4.5)$ & $0(0.0)$ & $0(0.0)$ & $46(5.7)$ & $276(3.8)$ \\
\hline
\end{tabular}

Notes: $* P<0.05, * * P<0.001$.

Abbreviations: IQR, interquartile range; $\mathrm{CCl}$, Charlson comorbidity index; NTD, new Taiwan dollar.

for type 2 diabetes patients for whom lifestyle change alone was not sufficient to achieve blood glucose targets; and in the later studied years, it was changed to metformin in patients typically obese with an estimated glomerular filtration rate $($ GFR) $>30 \mathrm{~mL} / \mathrm{min}$. Meglitinides are used in patients who have an allergy to sulfonylurea, in elderly who are unable to use insulin, and in whom the goal of avoiding hypoglycemia is important; it is also indicated in patients with decreased GFR or renal failure since it has little renal clearance. Acarbose is indicated for patients with postprandial hyperglycemia because it slows absorption of glucose. In older patients, acarbose may also increase insulin sensitivity. Insulin monotherapy is 
typically chosen as a first-line therapy, particularly in patients presenting with hemoglobin $\mathrm{A}_{1 \mathrm{c}}>10 \%$, fasting plasma glucose $>13.9 \mathrm{mmol} / \mathrm{L}(250 \mathrm{mg} / \mathrm{dL})$, random glucose consistently $>16.7 \mathrm{mmol} / \mathrm{L}(300 \mathrm{mg} / \mathrm{dL})$, or ketonuria.

\section{Follow-up and major outcomes}

The index date was defined as the date of initial prescription of a hypoglycemic agent. All patients were followed up until an incidence of cancer development, dropout from the insurance program, death, or until December 31, 2008.

To avoid protopathic bias (reverse causation) in this cohort study, patients developing cancer within the first year of follow-up were excluded from further study and the person-time was censored at the date of cancer diagnosis.

In addition to evaluating the overall incidence of cancer as the main outcome, the present study also evaluated the incidence of urinary bladder cancer (ICD-9-CM code 188), kidney and cancer of other urinary organs (189.x), colorectal cancer (CRC; 153 and 154), lung cancer (162), hepatocellular carcinoma (155.0), and breast cancer (174-175).

\section{Statistical analyses}

Continuous variables, including age, follow-up duration, and CCI for each group were presented as means with standard deviations or medians with interquartile ranges (IQR). The Kruskal-Wallis test was used to compare values within groups. Comparisons of categorical variables were performed using Pearson's chi-square test. Cumulative cancer incidence curves for each group were drawn using the Kaplan-Meier method. The log-rank test was used to compare cumulative cancer incidence between groups. Finally, unadjusted and multivariable-adjusted hazard ratios (HR), with corresponding 95\% confidence intervals (CI), for the development of cancer between the metformin group and other groups was estimated using Cox proportional hazards models adjusted for age, sex, CCI, smoking-related diagnoses, alcohol use disorders, morbid obesity, pancreatitis, hypertension, monthly household income, and urbanization level. Two-sided $P$-values $<0.05$ were required to reject null hypotheses. Statistical analyses were performed using the IBM ${ }^{\circledR}$ SPSS ${ }^{\circledR}$ Statistics version 22 (IBM Corporation, Armonk, NY, USA).

\section{Results}

With an overall median follow-up duration of 2.5 years (IQR, 3.6 years), 124 patients developed cancer in the metformin group, whereas 367 patients in the sulfonylurea group, 49 in the insulin group, 6 in the acarbose group, and 3 in the meglitinide group developed cancer.
The overall median age at baseline was 62.6 years (IQR 20.4). Patients in the metformin group were the youngest with a median age of 60.6 years (19.5), whereas those in the insulin group were the oldest with a median age of 70.6 years (22.5). There were more men than women in the study cohort, with a ratio of 1.2. The median number of comorbidities in this cohort, represented by CCI values, was 1.0 (2.0) (Table 1).

Overall, $16.5 \%$ of all patients had at least one smokingrelated diagnosis, 3.2\% had an alcohol use disorder, and morbid obesity was detected in $1.6 \%$ of all patients (Table 1 ). In this cohort, $64 \%$ of patients had hypertension, $43.7 \%$ had hyperlipidemia, and $1.4 \%$ had pancreatitis (Table 1 ). The assessment of the socioeconomic status according to monthly household income distribution demonstrated that nearly $70 \%$ of all patients came from lower income families (Table 1). The overall median follow-up period was 2.5 years (IQR 3.6 years), with $57.3 \%$ of patients followed up for less than 3 years and $11.7 \%$ of patients followed up for more than 7 years (Table 1$)$.

Using the metformin group as the comparator, multivariableadjusted Cox's models demonstrated that the adjusted HR of cancer was 1.36 in the sulfonylurea group (95\% CI, 1.11-1.67, $P=0.003)$. Figure 2 shows the cumulative cancer incidence in the sulfonylurea monotherapy group compared with metformin monotherapy. No significant differences in the risk of cancer development, according to calculated adjusted HRs, were observed between metformin and other groups (Table 2). The adjusted HR of cancer development compared with the metformin group was $0.54(95 \% \mathrm{CI}, 0.17-1.70)$ for the

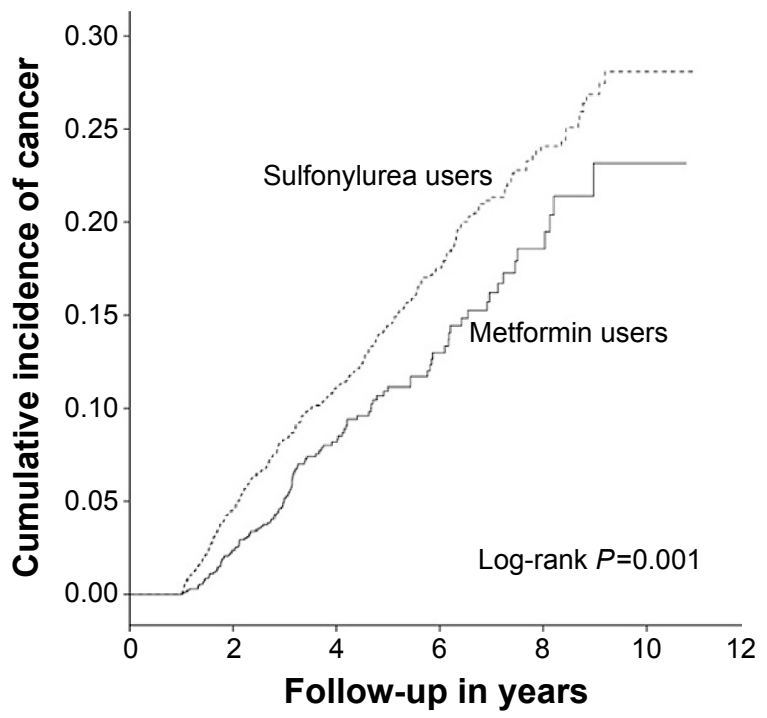

Figure 2 Cumulative incidence of cancer in the sulfonylurea monotherapy group demonstrating an adjusted $\mathrm{HR}$ of 1.36 with a $95 \% \mathrm{Cl}$ at I.I I-I.67 compared with metformin monotherapy.

Abbreviations: $\mathrm{HR}$, hazard ratio; $\mathrm{Cl}$, confidence interval. 
Table 2 Cox's regression model-derived HR for the incidence of cancer according to antidiabetic monotherapy

\begin{tabular}{|c|c|c|c|c|}
\hline Hypoglycemic & Number of patients & Cancer incidence & Unadjusted HR (95\% Cl) & Adjusted $\mathrm{HR}^{\mathrm{a}}(95 \% \mathrm{Cl})$ \\
\hline Metformin & 2,223 & 124 & 1 & 1 \\
\hline Sulfonylureas & 3,965 & 367 & $1.39(1.13-1.71)^{*}$ & $1.36(1.11-1.67)^{* *}$ \\
\hline Meglitinides & 128 & 3 & $0.62(0.20-1.95)$ & $0.54(0.17-1.70)$ \\
\hline Acarbose & 150 & 6 & $0.89(0.39-2.02)$ & $0.93(0.4 \mathrm{I}-2.12)$ \\
\hline Insulin & 806 & 49 & $1.18(0.85-1.65)$ & $1.05(0.74-1.48)$ \\
\hline
\end{tabular}

Notes: $* P=0.002$. ${ }^{*} * P=0.003$. a Multivariable Cox's regression model depicting HRs adjusted for age, sex, CCl, smoking-related diagnoses, alcohol use disorders, morbid obesity, pancreatitis, hypertension, hyperlipidemia, monthly household income, and urbanization level.

Abbreviations: $\mathrm{Cl}$, confidence interval; $\mathrm{HR}$, hazard ratio; $\mathrm{CCl}$, Charlson comorbidity index.

meglitinide group, $0.93(0.41-2.12)$ for the acarbose group, and $1.05(0.74-1.48)$ for the insulin group.

Subgroup analysis of the risk of selected cancer types between the metformin group and sulfonylurea group was performed using a Cox's regression model. In an unadjusted Cox's model, a significantly greater association between sulfonylurea monotherapy and an increased risk of CRC (HR 1.83, 95\% CI, 1.09-3.08), lung cancer (HR 1.81, 95\% CI, 1.04-3.15), and hepatocellular carcinoma (HR 1.73, 95\% CI, 1.01-2.98) development was observed compared with metformin monotherapy. After adjusting for age, sex, CCI, smoking-related diagnoses, alcohol use disorders, morbid obesity, pancreatitis, hypertension, hyperlipidemia, monthly household income, and urbanization level, the multivariableadjusted Cox's model demonstrated adjusted HRs for specific cancer types; 1.94 for CRC $(95 \% \mathrm{CI}, 1.15-3.27 ; P<0.05)$ and 1.76 for lung cancer $(95 \% \mathrm{CI}, 1.00-3.07 ; P<0.05$, Table 3$)$. Figure 3 shows the subgroup analysis of the cumulative incidence of CRC in the sulfonylurea monotherapy group compared with the metformin monotherapy group. Figure 4 shows the subgroup analysis of the cumulative incidence of lung cancer in the sulfonylurea monotherapy group compared with the metformin monotherapy group.

As to the differential cancer risks by different average defined daily dose (DDD) of metformin, when compared to an average DDD of $\leq 0.25$ (equivalent to $\leq 500 \mathrm{mg} / \mathrm{d}$ ) of metformin, our Cox model, adjusted for similar covariates as the other models, established that there was markedly reduced cancer risk in terms of adjusted HR at $0.20(95 \%$ CI, 0.10-0.40; $P<0.001$; Table 4).

\section{Discussion}

This population-based retrospective cohort study in adult patients with new-onset type 2 diabetes receiving antidiabetic monotherapy rejects the null hypothesis that metformin confers the same risk of subsequent cancer development as other groups of hypoglycemic drugs. In fact, this study demonstrates that sulfonylurea monotherapy is associated with a statistically significant increased risk of cancer development, with an adjusted HR of 1.36 .

Monotherapy for new-onset type $2 \mathrm{DM}$ is common because it is recommended by widely accepted clinical practice guidelines. However, recruiting adequate numbers of patients on prolonged monotherapy to cohort studies without crossing over to other agents is challenging because increasing numbers of patients are likely to require add-on combination therapy or crossover to other antidiabetic drugs. Our study is one of the very few cohort studies that have evaluated the risk of cancer development in diabetic patients on persistent monotherapy. Pharmacoepidemiologic study designs are particularly susceptible to confounding factors, with combination therapy and crossover being the two main confounders. In this database, approximately $14 \%$ of patients were persistently receiving

Table 3 HRs for specific cancer types with the use of SU and metformin

\begin{tabular}{lllll}
\hline Cancer type & Metformin & SU & Unadjusted HR (95\% CI) & Adjusted HR (95\% CI) \\
\hline Colorectum & 18 & 69 & $1.83(1.09-3.08)^{*}$ & $1.94(1.15-3.27)^{*}$ \\
Lung cancer & 16 & 59 & $1.81(1.04-3.15)^{*}$ & $1.76(1.00-3.07)^{*}$ \\
Hepatocellular carcinoma & 17 & 60 & $1.73(1.01-2.98)^{*}$ & $1.63(0.94-2.80)$ \\
Breast & 6 & 14 & $1.12(0.43-2.92)$ & $1.25(0.47-3.31)$ \\
Bladder cancer & 7 & 15 & $1.01(0.4 I-2.48)$ & $1.01(0.4 I-2.5 I)$ \\
Kidney + upper urinary tract cancer & 3 & 15 & $2.52(0.73-8.72)$ & $2.45(0.70-8.53)$ \\
\hline
\end{tabular}

Notes: $* P<0.05$. Patients could have more than one cancer in this analysis. Adjusted $\mathrm{HRs}$ were derived after adjustment for age, sex, $\mathrm{CCl}$, smoking-related diagnoses, alcohol use disorders, morbid obesity, pancreatitis, hypertension, hyperlipidemia, monthly household income, and urbanization level.

Abbreviations: $\mathrm{SU}$, sulfonylureas; $\mathrm{HR}$, hazard ratio; $\mathrm{Cl}$, confidence interval; $\mathrm{CCl}$, Charlson comorbidity index. 


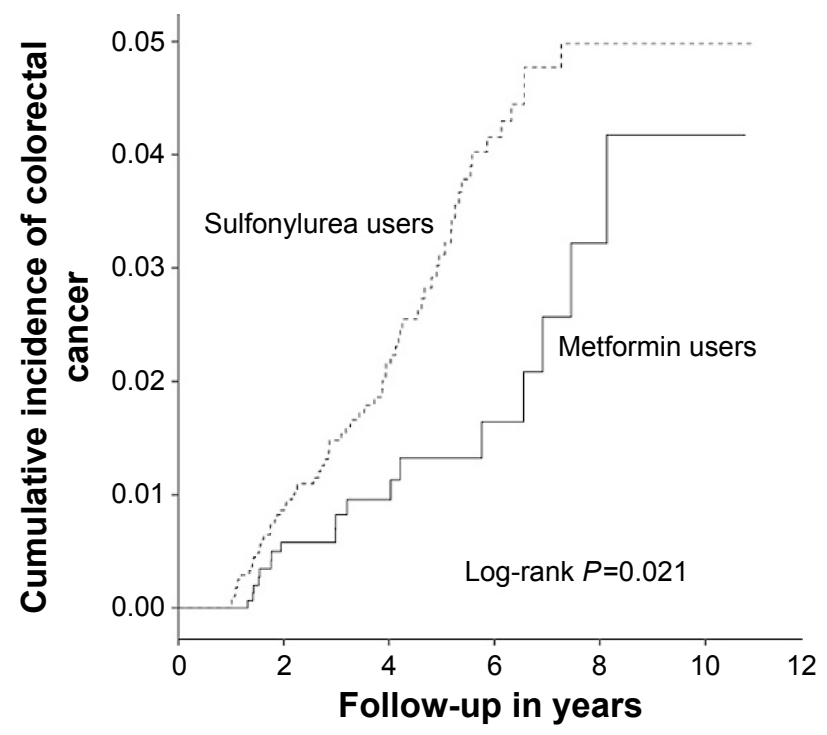

Figure 3 Subgroup analysis of the cumulative incidence of colorectal cancer in the sulfonylurea monotherapy group demonstrating an adjusted HR of I.94 with a 95\% confidence interval at I.I5-3.27 compared with the metformin monotherapy group.

Abbreviations: $\mathrm{HR}$, hazard ratio; $\mathrm{Cl}$, confidence interval.

antidiabetic monotherapy during the follow-up period of this longitudinal study. This allows an assessment of the exact proportion of real-world patients receiving monotherapy over an extended period of time.

Over the past decade, population-based observational studies have shown that metformin use is associated with a reduced risk of cancer devlopment. ${ }^{4}$ The magnitude of risk reduction was estimated as approximately $30 \%$ in a recent meta-analysis of cohort studies. ${ }^{7}$ The same meta-analysis

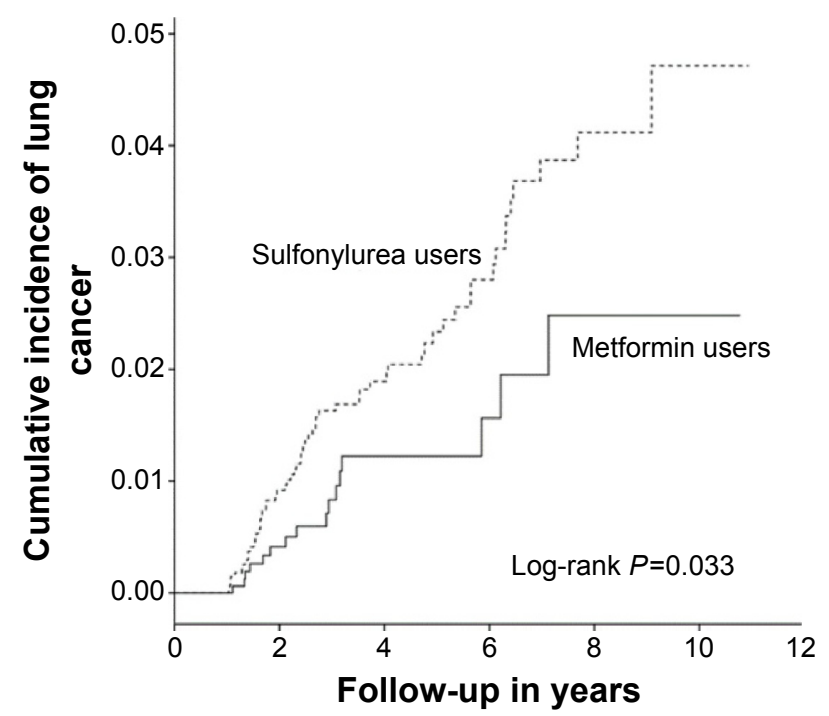

Figure 4 Subgroup analysis of the cumulative incidence of lung cancer in the sulfonylurea monotherapy group demonstrating an adjusted $\mathrm{HR}$ of $\mathrm{I} .76$ with a $95 \%$ $\mathrm{Cl}$ at I.00-3.07 compared with the metformin monotherapy group. Abbreviations: $\mathrm{HR}$, hazard ratio; $\mathrm{Cl}$, confidence interval. pooled cohort studies to demonstrate that sulfonylurea use is associated with an increase in all-cancer risk, with an HR of 1.55. ${ }^{7}$ Our population-based study of East Asian adults with type 2 diabetes corroborates these previous findings of an increased risk of cancer development with the use of sulfonylureas compared with metformin. We were also able to show in this study that a higher dose of metformin when compared to lower daily dosage was associated with markedly reduced cancer risk (Table 4).

Our understanding of the mechanisms underlying the anticancer effect of metformin has recently evolved. AMPK activation has previously been shown to stimulate downstream mTOR inhibition, leading to apoptosis in cancer-initiating and cancer stem cells in a number of malignant solid tumors types. More recently, it has become apparent that direct immune-mediated effects on $\mathrm{CD} 8^{+} \mathrm{T}$-cells protect $\mathrm{CD} 8^{+}$tumor-infiltrating lymphocytes from apoptosis, eventually resulting in tumor growth inhibition. ${ }^{23}$ Nonetheless, the mechanisms underlying the increased risk of subsequent cancer development with sulfonylurea monotherapy remain unknown. The difference in the risk of cancer development is most likely attributed to the beneficial risk reduction effect of metformin.

Our subgroup analysis of selected cancer types demonstrated that sulfonylurea monotherapy was associated with an increased risk of CRC and lung cancer development. In CRC, a meta-analysis of the association between metformin use and the risk of CRC development found that metformin therapy was associated with a significantly lower risk of CRC development, representing a $37 \%$ reduction in relative risk $(\mathrm{RR}=0.63$ [95\% CI, 0.47-0.84]; $P=0.002) .{ }^{8}$ However, other retrospective observational studies failed to detect a significant risk reduction in CRC development with metformin use. ${ }^{11,13}$ In lung cancer, a nested case-control population-based study failed to detect a significant risk reduction in "ever users" of metformin. ${ }^{15}$ However, a meta-analysis of metformin therapy and lung cancer risk found that metformin use appears to be associated with lower risk of lung cancer development. ${ }^{24} \mathrm{~A}$ direct comparison between two observational studies was not generally feasible because of variations in study design, confounding factors, and statistical models used. Prospective randomized controlled trials are required to definitively compare the risk of cancer development with metformin monotherapy to other antidiabetic monotherapies.

Using the same criteria and method for controlling confounding factors, no difference in the risk of cancer development was observed between insulin monotherapy and metformin monotherapy in our study (adjusted HR $=1.05$; $95 \% \mathrm{CI}, 0.74-1.48)$. This result is noteworthy because several 
Table 4 Cancer risk by different average DDD of metformin monotherapy

\begin{tabular}{|c|c|c|c|c|c|}
\hline Average DDD ${ }^{a}$ & Average daily dosage (mg) & $\mathbf{N}$ & Person-year & Incident cancer & Adjusted $\mathrm{HR}^{\mathrm{b}}(95 \% \mathrm{Cl})$ \\
\hline$\leq 0.25$ & $\leq 500$ & 1,522 & $4,854.6$ & 97 & 1 \\
\hline$>0.25$ & $>500$ & 701 & I,822.4 & 27 & $0.20(0.10-0.40)^{*}$ \\
\hline
\end{tabular}

Notes: ${ }^{*} P<0.001 .{ }^{2}$ Average DDD defined as average cumulative DDD during the follow-up period. (During the follow-up period, patients might not take metformin every

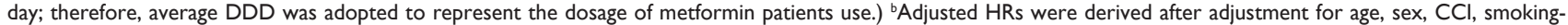
related diagnoses, alcohol use disorders, morbid obesity, pancreatitis, hypertension, hyperlipidemia, monthly household income, and urbanization level.

Abbreviations: DDD, defined daily dose; $\mathrm{Cl}$, confidence interval; $\mathrm{HR}$, hazard ratio; $\mathrm{N}$, patient number; $\mathrm{CCl}$, Charlson comorbidity index.

studies have suggested that insulin therapy is associated with an increased risk of cancer development. ${ }^{25-27}$

\section{Strengths and limitations}

In contrast to a questionnaire- or an interview-based methodology, recall bias and misclassification bias were avoided by our study design that provided good case ascertainment because every participant had a confirmed ICD-9-CM code for type 2 diabetes while concurrently receiving an oral hypoglycemic agent. Crossover was not allowed in this longitudinal study; therefore, there was no effect of allocation bias. Protopathic bias (reverse causation) was also avoided in this study because we excluded cancers that occurred prior to the index date and within the first year of the follow-up period. Participants were followed up for the entire study period with no cases excluded from the final analysis. We believed confounders were optimally controlled in this study because Cox's models were used to adjust for medical comorbidities according to CCI, smoking-related diagnoses, alcohol use disorders, monthly household income as a proxy for socioeconomic group, and urbanization strata as a proxy of geographic lifestyle.

Nevertheless, this study had certain potential limitations. First, the definition of the target population of diabetic patients receiving long-term monotherapeutic agent substantially decreased the number of included patients, thus resulting in inadequate statistical power to fully evaluate the groups of patients receiving glitazone, meglitinide, or acarbose. Similar to other pharmacoepidemiologic studies utilizing claims database, body mass index information and measures of glycemic control such as plasma hemoglobin $\mathrm{A}_{1 \mathrm{c}}$ concentrations were not available for analysis. However, because of an elevated risk of almost $40 \%$ in patients receiving sulfonylurea, the direction of risk is highly unlikely to change with the addition of this missing information.

\section{Conclusion}

This population-based retrospective cohort study found that sulfonylurea monotherapy may be associated with a $36 \%$ increase in the risk of cancer development compared with metformin monotherapy. These results may be alternatively interpreted as a reduced risk of future cancer development in patients receiving metformin compared with those receiving sulfonylureas. Higher dose of metformin at $>0.25$ average DDD (equivalent to $>500 \mathrm{mg} / \mathrm{d}$ ) when compared with lower dosage of metformin is associated with $80 \%$ reduced risk of cancer development.

\section{Acknowledgments}

The authors are grateful to the Taiwan National Health Research Institute for providing the data set for this study. The interpretation and conclusions contained herein do not represent those of the aforementioned institution. This work was partly supported by grants from the Ministry of Science and Technology of Taiwan (MOST 103-2221-E-468-022) and Asia University Taiwan (103-ASIA-04). The funders had no role in study design, data collection, data analysis, preparation of the manuscript, or decision to publish.

\section{Disclosure}

The authors have no competing interests. The authors declare neither potential nor perceived conflict of interests.

\section{References}

1. Danaei G, Finucane MM, Lu Y, et al. National, regional, and global trends in fasting plasma glucose and diabetes prevalence since 1980: systematic analysis of health examination surveys and epidemiological studies with 370 country-years and 2.7 million participants. Lancet. 2011;378(9785):31-40.

2. Vigneri P, Frasca F, Sciacca L, Pandini G, Vigneri R. Diabetes and cancer. Endocr Relat Cancer. 2009;16(4):1103-1123.

3. Nathan DM, Buse JB, Davidson MB, et al. Medical management of hyperglycemia in type 2 diabetes: a consensus algorithm for the initiation and adjustment of therapy: a consensus statement of the American Diabetes Association and the European Association for the Study of Diabetes. Diabetes Care. 2009;32(1):193-203.

4. Evans JM, Donnelly LA, Emslie-Smith AM, Alessi DR, Morris AD. Metformin and reduced risk of cancer in diabetic patients. $B M J$. 2005;330(7503):1304-1305.

5. Libby G, Donnelly LA, Donnan PT, Alessi DR, Morris AD, Evans JM. New users of metformin are at low risk of incident cancer: a cohort study among people with type 2 diabetes. Diabetes Care. 2009;32(9): $1620-1625$.

6. Ruiter R, Visser LE, van Herk-Sukel MP, et al. Lower risk of cancer in patients on metformin in comparison with those on sulfonylurea derivatives: results from a large population-based follow-up study. Diabetes Care. 2012;35(1):119-124. 
7. Thakkar B, Aronis KN, Vamvini MT, Shields K, Mantzoros CS. Metformin and sulfonylureas in relation to cancer risk in type II diabetes patients: a meta-analysis using primary data of published studies. Metabolism. 2013;62(7):922-934.

8. Zhang ZJ, Zheng ZJ, Kan H, et al. Reduced risk of colorectal cancer with metformin therapy in patients with type 2 diabetes: a meta-analysis. Diabetes Care. 2011;34(10):2323-2328.

9. Shaw RJ, Lamia KA, Vasquez D, et al. The kinase LKB1 mediates glucose homeostasis in liver and therapeutic effects of metformin Science. 2005;310(5754):1642-1646.

10. Azoulay L, Dell'Aniello S, Gagnon B, Pollak M, Suissa S. Metformin and the incidence of prostate cancer in patients with type 2 diabetes. Cancer Epidemiol Biomarkers Prev. 2011;20(2):337-344.

11. Bodmer M, Becker C, Meier C, Jick SS, Meier CR. Use of metformin is not associated with a decreased risk of colorectal cancer: a case-control analysis. Cancer Epidemiol Biomarkers Prev. 2012;21(2):280-286.

12. Mamtani R, Pfanzelter N, Haynes K, et al. Incidence of bladder cancer in patients with type 2 diabetes treated with metformin or sulfonylureas. Diabetes Care. 2014;37(7):1910-1917.

13. Qiu H, Rhoads GG, Berlin JA, Marcella SW, Demissie K. Initial metformin or sulphonylurea exposure and cancer occurrence among patients with type 2 diabetes mellitus. Diabetes Obes Metab. 2013;15(4): 349-357.

14. Simo R, Plana-Ripoll O, Puente D, et al. Impact of glucose-lowering agents on the risk of cancer in type 2 diabetic patients. The Barcelona case-control study. PLoS One. 2013;8(11):e79968.

15. Smiechowski BB, Azoulay L, Yin H, Pollak MN, Suissa S. The use of metformin and the incidence of lung cancer in patients with type 2 diabetes. Diabetes Care. 2013;36(1):124-129.

16. van Staa TP, Patel D, Gallagher AM, de Bruin ML. Glucose-lowering agents and the patterns of risk for cancer: a study with the General Practice Research Database and secondary care data. Diabetologia. 2012;55(3):654-665.

17. Walker EJ, Ko AH, Holly EA, Bracci PM. Metformin use among type 2 diabetics and risk of pancreatic cancer in a clinic-based case-control study. Int J Cancer. 2015;136(6):E646-E653.
18. Tsilidis KK, Capothanassi D, Allen NE, et al. Metformin does not affect cancer risk: a cohort study in the U.K. Clinical Practice Research Datalink analyzed like an intention-to-treat trial. Diabetes Care. 2014;37(9):2522-2532.

19. Kok VC, Horng JT, Chang WS, Hong YF, Chang TH. Allopurinol therapy in gout patients does not associate with beneficial cardiovascular outcomes: a population-based matched-cohort study. PLoS One. 2014;9(6):e99102.

20. Kok VC, Horng JT, Huang JL, et al. Population-based cohort study on the risk of malignancy in East Asian children with juvenile idiopathic arthritis. BMC Cancer. 2014;14:634.

21. Kok VC, Horng JT, Lin HL, Chen YC, Chen YJ, Cheng KF. Gout and subsequent increased risk of cardiovascular mortality in non-diabetics aged 50 and above: a population-based cohort study in Taiwan. BMC Cardiovasc Disord. 2012;12:108.

22. Kok VC, Tsai HJ, Su CF, Lee CK. The risks for ovarian, endometrial, breast, colorectal, and other cancers in women with newly diagnosed endometriosis or adenomyosis: a population-based study. Int J Gynecol Cancer. 2015;25(6):968-976.

23. Eikawa S, Nishida M, Mizukami S, Yamazaki C, Nakayama E, Udono H. Immune-mediated antitumor effect by type 2 diabetes drug, metformin. Proc Natl Acad Sci U S A. 2015;112(6):1809-1814.

24. Zhang ZJ, Bi Y, Li S, et al. Reduced risk of lung cancer with metformin therapy in diabetic patients: a systematic review and meta-analysis. Am J Epidemiol. 2014;180(1):11-14.

25. Bu WJ, Song L, Zhao DY, Guo B, Liu J. Insulin therapy and the risk of colorectal cancer in patients with type 2 diabetes: a meta-analysis of observational studies. Br J Clin Pharmacol. 2014;78(2):301-309.

26. Currie CJ, Poole CD, Gale EA. The influence of glucose-lowering therapies on cancer risk in type 2 diabetes. Diabetologia. 2009;52(9): 1766-1777.

27. Newton CC, Gapstur SM, Campbell PT, Jacobs EJ. Type 2 diabetes mellitus, insulin-use and risk of bladder cancer in a large cohort study. Int J Cancer. 2013;132(9):2186-2191.
Therapeutics and Clinical Risk Management

\section{Publish your work in this journal}

Therapeutics and Clinical Risk Management is an international, peerreviewed journal of clinical therapeutics and risk management, focusing on concise rapid reporting of clinical studies in all therapeutic areas outcomes, safety, and programs for the effective, safe, and sustained use of medicines. This journal is indexed on PubMed Central, CAS,

\section{Dovepress}

EMBase, Scopus and the Elsevier Bibliographic databases. The manuscript management system is completely online and includes a very quick and fair peer-review system, which is all easy to use. Visit http://www.dovepress.com/testimonials.php to read real quotes from published authors. 